ORIGINAL PAPER

\title{
Molecular classification of glioblastoma based on IMMUNOHISTOCHEMICAL EXPRESSION OF EGFR, PDGFRA, NF1, IDH1, P53 AND PTEN PROTEINS
}

\author{
Sylwia Jankowska ${ }^{1}$, Magdalena Lewandowska ${ }^{1}$, Marta Masztalewicz $^{2}$, Leszek Sagan ${ }^{3}$, \\ Przemyseaw Nowacki ${ }^{2}$, Elżbieta Urasińska ${ }^{1}$
}

\begin{abstract}
${ }^{1}$ Department of Pathology, Pomeranian Medical University, Szczecin, Poland
${ }^{2}$ Department of Neurology, Pomeranian Medical University, Szczecin, Poland

${ }^{3}$ Department of Neurosurgery, Pomeranian Medical University, Szczecin, Poland
\end{abstract}

\begin{abstract}
Glioblastoma (GBM) is the most common and most aggressive primary tumor of the central nervous system. Current GBM treatments have low effectiveness. This is mainly due to the high degree of heterogeneity of GBM tumors. Despite similarities in the classic microscopic image, these tumors differ significantly in molecular terms. The aim of the study was to classify GBM tumors into one of four molecular types based on the immunohistochemical expression of EGFR, PDGFRA, NF1, IDH1, p53 and PTEN proteins and find the association between individual glioma molecular types and prognostic clinical and morphological parameters. From the group of 162 patients the classical molecular type of tumor was observed in $17(10 \%)$ patients, in $23(14 \%)$ the tumor was mesenchymal, in $32(20 \%)$ proneural, and in $90(56 \%)$ neural. No significant relationship was observed between the molecular type of GBM tumors and the studied clinical and morphological parameters of prognostic significance. There were also no statistically significant correlations between the GBM tumor molecular type and survival, both in terms of overall survival and relapse-free survival. Analyzing the impact of all prognostic variables and molecular type of GBM on the probability of overall survival, statistically significant relationships were found.
\end{abstract}

Key words: glioblastoma, EGFR, PDGFRA, NF1, IDH1.

\section{Introduction}

Glioblastoma (GBM) is the most common primary tumor of the central nervous system. It is the neoplasm with the highest aggressiveness and the worst prognosis among all tumors of glial origin; the median survival is 14 months, and the 5 -year survival is $4-5 \%[1,2]$. Current GBM treatments include surgical resection, radiation therapy (RTH), and chemotherapy $(\mathrm{CTH})$ with temozolomide. However, the persistently high mortality rate due to GBM indicates low effectiveness of the above-mentioned therapies. This is mainly due to the high degree of heterogeneity of GBM tumors. Despite similarities in the classic microscopic image, these tumors differ significantly in molecular terms. This results in their different prognosis and different sensitivity to the treatment used. Classic prognostic factors used in GBM (age at diagnosis, patient's gender, tumor diameter, tumor location, primary vs. secondary nature of the tumor, monofocal vs. multifocal tumor, extent of surgical resection, use of CTH or RTH, overall survival and relapse-free survival) do not always allow one to fully predict the course of the dis- 
ease. This is why the molecular profile of the tumor is more and more often assessed. Based on it, we can differentiate GBM tumors in terms of prognosis, clinical course and response to treatment, giving an opportunity for the individualization of the therapy in the future $[2,3,4,5]$. The current molecular classification of GBM distinguishes four molecular types of GBM tumors: classical, mesenchymal, proneural and neural. In the classical type of GBM, abnormalities in the EGFR gene are most pronounced among all molecular types. This gene codes the protein of the same name, which is a key growth factor involved in the pathogenesis of GBM tumors. The most common of the abnormalities mentioned above is the EGFRvIII mutation. This type also shows molecular changes in the following genes: CDKN2A, RB1, CDK4, CCDN2, NOTCH3, $J A G 1, L F N G, S M O, G A S 1, G L I 2$. The gene expression profile observed in this group recalls that typical of astrocytes. The lack of TP53 gene mutations results in their sensitivity to classic therapy based on the use of DNA-alkylating drugs in conjunction with radiation therapy. In the mesenchymal type of GBM, in which neoplastic cells resemble astroglial cells, the most common are mutations in the following genes: NF1, PTEN, AKT, MET, CHI3LI, TP53 and those belonging to the NF$\kappa \beta$ pathway (TRADD, RELB, TNFRSF1A). This kind of gene expression profile implies high sensitivity to aggressive chemo- and radiotherapy and angiogenesis inhibitors. Cells of proneural tumors are characterized by oligodendrocyte-like gene expression and mutations in the following genes: PDGFRA and PDGFRB, IDH1, p53, PIK3CA/ PIK3RI, HIF, CDKN1A, NKX2-2, OLIG2, SOX, DCX, DLL3, ASCL1 and TCF4. They do not respond to conventional treatment and are sensitive to PI3K, HIF and PDGFRA inhibitors. Moreover, this type often correlates with a young age of onset and a lower clinical stage at the time of diagnosis, and is also characteristic of secondary tumors. Cells of the neural type tumors resemble normal oligodendrocytes and astrocytes in molecular and morphological terms. They show the presence of less specific mutations within the genes: NEFL, GABRA1, SYT1, SLC12A5, and to a lesser extent TP53, which means that these tumors show limited sensitivity to classical methods of therapy $[6,7,8]$. In practice, however, gene expression testing methods are very expensive and used mainly for scientific purposes, which is why they are increasingly being replaced by immunohistochemical (IHC) methods $[9,10,11]$. In this study we decided to classify GBM tumors into one of four molecular types (classical, mesenchymal, proneural or neural) based on the expression of EGFR, PDGFRA, NF1, IDH1, p5 3 and PTEN proteins and try to find the associa- tion between individual glioma molecular types and prognostic clinical and morphological parameters, such as age at diagnosis, patient's gender, tumor diameter, tumor location, primary vs. secondary nature of the tumor, monofocal vs. multifocal tumor, extent of surgical resection, use of CTH or RTH, overall survival and relapse-free survival.

\section{Material and methods}

\section{Study group}

The study included a group of 162 unselected patients diagnosed with GBM based on the histopathological examination of postoperative material or biopsy of a brain tumor, who underwent treatment in the years 2008-2013 at the Department of Neurosurgery of the Pomeranian Medical University in Szczecin The study group was characterized according to the following clinical and morphological parameters: age at diagnosis, patient's gender, tumor diameter, tumor location, primary vs. secondary nature of the tumor, monofocal vs. multifocal tumor, extent of surgical resection, use of $\mathrm{CTH}$ or $\mathrm{RTH}$, overall survival and relapse-free survival. The study was approved by the Bioethics Committee of the Pomeranian Medical University. The clinical and morphological characteristics of the study group are presented in Table I.

\section{Immunohistochemical staining}

Tissue material fixed in $10 \%$ buffered formalin and embedded in paraffin was used for the study. The expression of EGFR, PDGFRA, NF1, IDH1, p53 and PTEN proteins was determined by immunohistochemistry using the following antibodies: monoclonal rabbit anti-EGFR antibody (clone 5B7, Ventana (ROCHE), No. 790-4347), polyclonal rabbit anti-PDGFRA antibody (LifeSpan BioSciences, No. LS-B2172), mouse anti-NF1 monoclonal antibody (McNFn27 clone, LifeSpan BioSciences, No. LS-B217), polyclonal rabbit anti-IDH1 antibody (LifeSpan BioSciences, No. LS-B3573), mouse anti-p53 monoclonal antibody (DO-7 clone, Dako, No. M7001), monoclonal mouse anti-PTEN antibody (GH2.1 clone, Dako, No. M3627). The preparations were dewaxed in an incubator (temperature $60^{\circ} \mathrm{C}$ ), then, in order to unmask the antigen, the preparations were heat treated at $96^{\circ} \mathrm{C}, \mathrm{pH}=6$. The sections cooled down to $65^{\circ} \mathrm{C}$ were incubated with peroxidase solution and then with the tested antibodies. Visualization of the antigen-antibody reaction was done with the following detection systems: LSAB + (Dako, No. K0679) for IDH1, PDGFRA and NF1, EnVision + (Dako, No. K4004/K4005) for p53 and PTEN and the ultraView Universal DAB Detection 
Kit (Ventana (ROCHE), No. 760-500) for EGFR. Slides were stained with Mayer's hematoxylin, dehydrated and covered with coverslips. The positive controls for the tested proteins were: normal skin for EGFR, normal breast tissue for PDGFRA, normal brain tissue for NF1, normal kidney for IDH1, colorectal cancer for p53, and endometrial cancer for PTEN. The negative control was the slides incubated with PBS instead of the antibody.

\section{Evaluation of protein expression}

The percentage of GBM cells expressing the tested proteins was calculated using virtual microscopy and computer image analysis in ImageScope. Material from 3 tissue cores taken from 3 different places within the neoplastic tumor was analyzed for each patient. The total number of cells analyzed from the material collected from one patient was thus the sum of the number of cells from 3 different tissue cores. For the EGFR and PDGFRA proteins, the level of their membrane expression was analyzed, for the $\mathrm{p} 53$ protein nuclear expression was analyzed, and for the remaining proteins cytoplasmic expression was analyzed. Each time the percentage of all positive cells, i.e. cells expressing a specific protein, was assigned to one of 6 groups: group $1=1-4 \%$, group $2=5-19 \%$, group $3=20-39 \%$, group $4=40-59 \%$, group $5=60-$ $79 \%$, group $6=80-100 \%$. Because the analyzed cells showed differences in the level of the positive intensity of the IHC reaction to the presence of a given protein. Each of them was assigned by the computer program to one of the 3 intensity levels: weakly positive $(1+)$, medium positive $(2+)$ and strongly positive $(3+)$. Based on the intensity of the IHC reaction $(1+, 2+, 3+)$ and the group of the percentage of cells positive for a given IHC reaction (groups 1-6), a unified index of the level of expression of the tested protein Quick score was introduced, according to the formula: QUICK SCORE $=\%$ positive cell group $\mathrm{x}$ IHC reaction intensity. Thus, the Quick scores ranged from 0 to 18. Due to the fact that the distribution of variables differed from normal, the median was each time assumed as the cut-off point for positive values. The cut-off points for the analyzed proteins were: $\mathrm{EGFR}=10$, PDGFRA $=6, \mathrm{NF} 1=6, \mathrm{IDH} 1=2$, $\mathrm{P} 53=1 . \mathrm{PTEN}=5$. Then, based on positive or negative IHC staining for the tested proteins, GBM tumors were classified into one from four molecular types: classical, mesenchymal, proneural or neural, according to the following profile: classical type: EGFR $(+)$, p53 (-), mesenchymal type: NF1 (-), PTEN $(+)$, p53 $(+)$, proneural type: PDGFRA $(+)$, IDH1 (+), p53 (+), neural type: established by excluding the above-mentioned combinations.
Table I. Characteristics of the study group $(\mathrm{n}=162)$

\begin{tabular}{|c|c|}
\hline \multicolumn{2}{|c|}{ Age at diagnosis $(\mathrm{n}=162)$} \\
\hline Minimum & 20 \\
\hline Maximum & 79 \\
\hline Average & $56.5 \pm 13.1$ \\
\hline Median & 59 \\
\hline \multicolumn{2}{|c|}{ Gender $(n=162)$} \\
\hline Female & $67(41 \%)$ \\
\hline Male & $95(59 \%)$ \\
\hline \multicolumn{2}{|c|}{ Tumor diameter $(\mathrm{cm})(\mathrm{n}=118)$} \\
\hline Minimum & 0.5 \\
\hline Maximum & 4.4 \\
\hline Average & $2.2 \pm 0.8$ \\
\hline Median & 2.3 \\
\hline \multicolumn{2}{|c|}{ Tumor location $(\mathrm{n}=162)$} \\
\hline Left-sided & $72(44 \%)$ \\
\hline Right-sided & $82(51 \%)$ \\
\hline Bilateral & $8(5 \%)$ \\
\hline \multicolumn{2}{|c|}{ Primary vs. secondary tumor $(\mathrm{n}=162)$} \\
\hline Primary & $158(98 \%)$ \\
\hline Secondary & $4(2 \%)$ \\
\hline \multicolumn{2}{|c|}{ Monofocal vs. multifocal tumor $(\mathrm{n}=162)$} \\
\hline Monofocal & $154(95 \%)$ \\
\hline Multifocal & $8(5 \%)$ \\
\hline \multicolumn{2}{|c|}{ Extent of surgical resection $(\mathrm{n}=162)$} \\
\hline Total & $99(61 \%)$ \\
\hline Partial & $63(39 \%)$ \\
\hline \multicolumn{2}{|c|}{ Chemotherapy ( $\mathrm{n}=162)$} \\
\hline Used & $33(20 \%)$ \\
\hline Not used & $129(80 \%)$ \\
\hline \multicolumn{2}{|c|}{ Radiation therapy $(\mathrm{n}=162)$} \\
\hline Used & $35(22 \%)$ \\
\hline Not used & $127(78 \%)$ \\
\hline \multicolumn{2}{|c|}{ Overall survival $(\mathrm{n}=162)$} \\
\hline Minimum & 4 \\
\hline Maximum & 1868 \\
\hline Average & $327.3 \pm 321.2$ \\
\hline Median & 235 \\
\hline \multicolumn{2}{|c|}{ Relapse free survival $(\mathrm{n}=39)$} \\
\hline Minimum & 21 \\
\hline Maximum & 1070 \\
\hline Average & $188.5 \pm 231.8$ \\
\hline Median & 109 \\
\hline
\end{tabular}



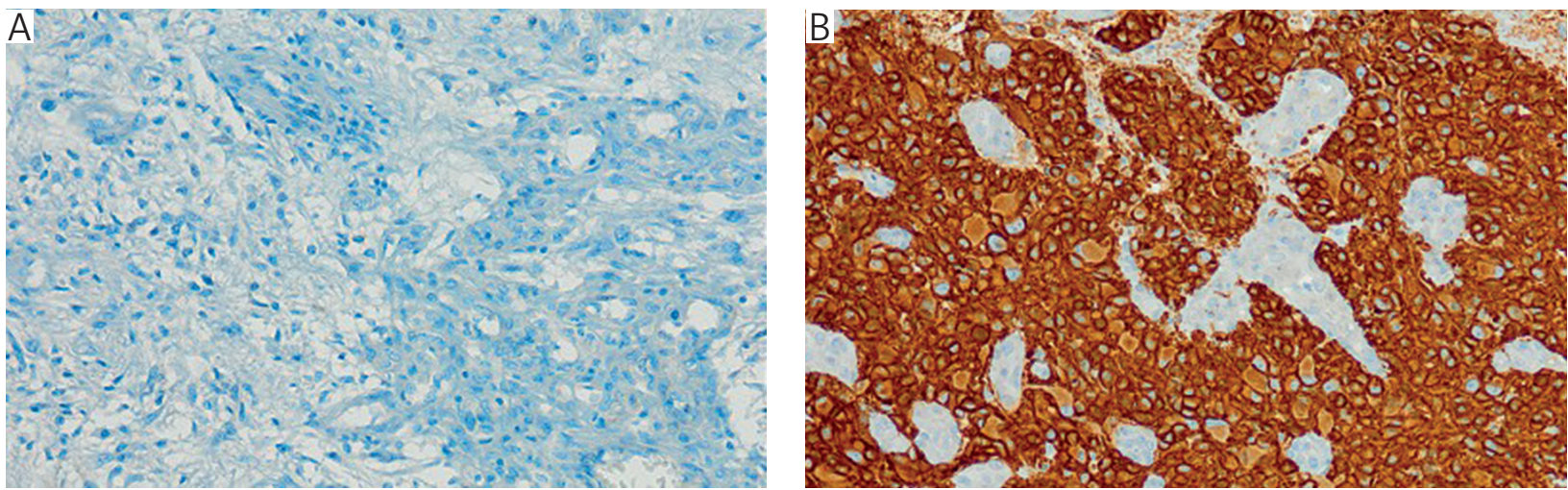

Fig. 1. IHC staining for EGFR in glioblastoma cells. Membrane expression. Magnification $20 \times$. A) Negative reaction; B) positive reaction $(3+)$
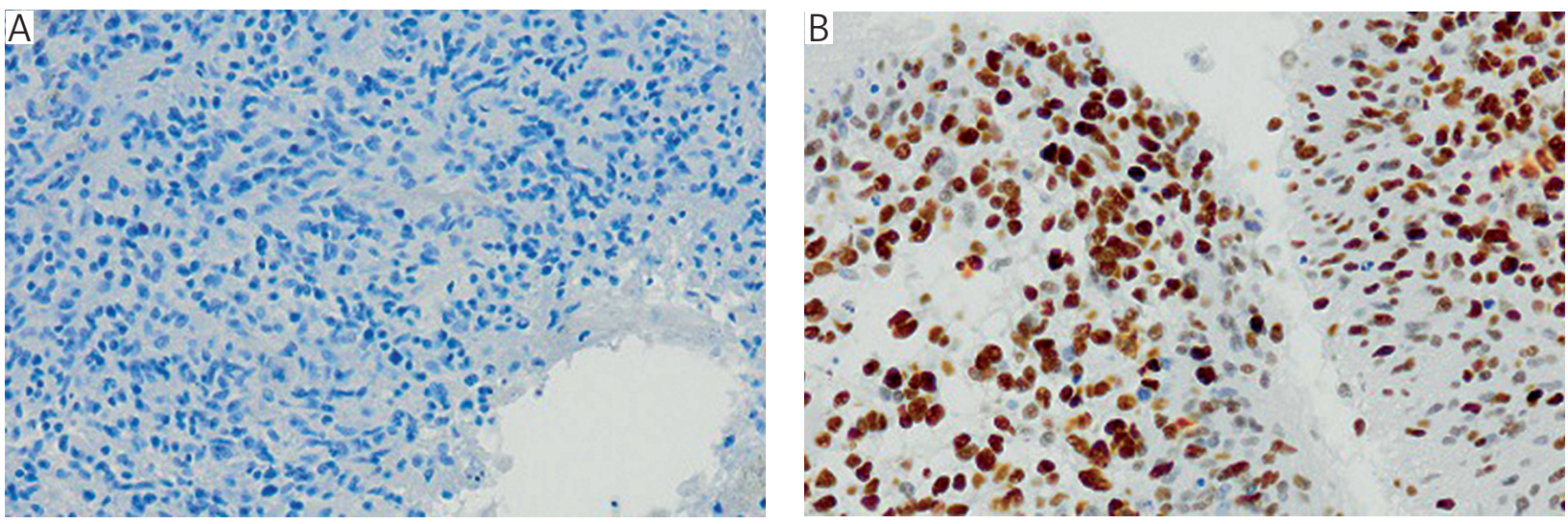

Fig. 2. IHC staining for p53 in glioblastoma cells. Nuclear expression. Magnification $20 \times$. A) Negative reaction; B) positive reaction $(3+)$

\section{Statistical analysis}

Statistical analysis was performed using the MedCalc program (version 19.2, Ostend, Belgium). The Shapiro-Wilk test was used to check the normality of the distribution. As the distribution of continuous variables deviated from the normal, the results were presented as medians, and the statistical analyses used non-parametric tests (Kruskal-Wallis, Mann-Whitney U, Spearman's rank correlations). Qualitative variables were characterized by numbers, and the $\chi^{2}$ test was used to assess the relationship between them. Survival analysis included univariate (Kaplan-Maier method) and multi-factor (Cox proportional hazards model) estimates. P-values $<0.05$ were considered statistically significant. If the results were not statistically significant, they were designated as NS (not significant).

\section{Results}

Examples of the IHC reactions of the analyzed proteins are presented in Figs. 1 and 2. After computer analysis of the morphological features and expression of the studied proteins in GBM tumor cells, they were assigned to one of 4 molecular types: classical, mesenchymal, proneural or neural. The classical type was found in $17(10 \%)$ patients, in $23(14 \%)$ the tumor was mesenchymal type, in $32(20 \%)$ proneural, and in $90(56 \%)$ neural. No significant relationship was observed between the molecular type of GBM tumors and the studied clinical and morphological parameters of prognostic significance, such as: age at diagnosis, patient's gender, tumor diameter, tumor location, primary vs. secondary nature of the tumor, monofocal vs. multifocal tumor, extent of surgical resection, use of CTH or RTH, overall survival and relapse-free survival. The lowest age of GBM onset was recorded in the proneural type (20 years), and the highest in the mesenchymal and neural types (79 years). The lowest median age at diagnosis was recorded in the classical GBM type (median $=52$ years; $\mathrm{SD}=6.5$ ), and the highest in the mesenchymal type (median $=63$ years; $S D=6$ ). Neural-type tumors were the most common among women and men, accounting for $54 \%$ of all tumors in women and $57 \%$ in men, respectively. The least numerous in both sexes were classical tumors (9\% in women, $12 \%$ in men). The highest median 
Table II. The relationship between tumor types and clinical and morphological parametres

\begin{tabular}{|c|c|c|c|c|}
\hline & \multicolumn{4}{|c|}{ MOLECULAR TYPE } \\
\hline & Classical & MESENCHYMAL & Proneural & Neural \\
\hline \multicolumn{5}{|l|}{ Age at diagnosis (years) $(n=162)$} \\
\hline Molecular types distribution & $17(10 \%)$ & $23(14 \%)$ & $32(20 \%)$ & $90(56 \%)$ \\
\hline Minimum & 31 & 36 & 20 & 28 \\
\hline Maximum & 71 & 79 & 78 & 79 \\
\hline Median & 52 & 63 & 60 & 595 \\
\hline $\mathrm{SD}$ & 6,6 & 6 & 7 & 7 \\
\hline $\mathrm{p}$-value & \multicolumn{4}{|c|}{0,19} \\
\hline \multicolumn{5}{|l|}{ Gender $(n=162)$} \\
\hline Molecular types distribution & $17(10 \%)$ & $23(14 \%)$ & $32(20 \%)$ & $90(56 \%)$ \\
\hline Female & $6(9 \%)$ & $10(15 \%)$ & $15(22 \%)$ & $36(54 \%)$ \\
\hline Male & $11(12 \%)$ & $13(14 \%)$ & $17(18 \%)$ & $54(57 \%)$ \\
\hline p-value & \multicolumn{4}{|c|}{0.86} \\
\hline \multicolumn{5}{|l|}{ Tumor diameter $(\mathrm{cm})(\mathrm{n}=118)$} \\
\hline Molecular types distribution & $13(11 \%)$ & $12(10 \%)$ & $22(19 \%)$ & $71(60 \%)$ \\
\hline Minimum & 1.1 & 0.9 & 0.7 & 0.5 \\
\hline Maximum & 3.8 & 3.6 & 3.3 & 4.4 \\
\hline Median & 2.2 & 1.95 & 2.55 & 2.2 \\
\hline SD & 0.28 & 0.55 & 0.35 & 0.4 \\
\hline $\mathrm{p}$-value & \multicolumn{4}{|c|}{0.36} \\
\hline \multicolumn{5}{|l|}{ Tumor location $(\mathrm{n}=162)$} \\
\hline Molecular types distribution & $17(10 \%)$ & $23(14 \%)$ & $32(20 \%)$ & $90(56 \%)$ \\
\hline Left-sided & $8(11 \%)$ & $11(15 \%)$ & $15(21 \%)$ & $38(53 \%)$ \\
\hline Right-sided & $9(11 \%)$ & $10(12 \%)$ & $15(18 \%)$ & $48(59 \%)$ \\
\hline Bilateral & $0(0 \%)$ & $2(25 \%)$ & $2(25 \%)$ & $4(50 \%)$ \\
\hline $\mathrm{p}$-value & \multicolumn{4}{|c|}{0.89} \\
\hline \multicolumn{5}{|c|}{ Primary vs. secondary tumor $(\mathrm{n}=162)$} \\
\hline Molecular types distribution & $17(10 \%)$ & $23(14 \%)$ & $32(20 \%)$ & $90(56 \%)$ \\
\hline Primary & $17(11 \%)$ & $23(15 \%)$ & $31(20 \%)$ & $87(55 \%)$ \\
\hline Secondary & $0(0 \%)$ & $0(0 \%)$ & $1(25 \%)$ & $3(75 \%)$ \\
\hline p-value & \multicolumn{4}{|c|}{0.72} \\
\hline \multicolumn{5}{|c|}{ Monofocal vs. multifocal tumor $(\mathrm{n}=162)$} \\
\hline Molecular types distribution & $17(10 \%)$ & $23(14 \%)$ & $32(20 \%)$ & $90(56 \%)$ \\
\hline Monofocal & $16(10 \%)$ & $21(14 \%)$ & $31(20 \%)$ & $86(56 \%)$ \\
\hline Multifocal & $1(12.5 \%)$ & $2(25 \%)$ & $1(12.5 \%)$ & $4(50 \%)$ \\
\hline p-value & \multicolumn{4}{|c|}{0.8} \\
\hline \multicolumn{5}{|c|}{ Extent of surgical resection $(\mathrm{n}=162)$} \\
\hline Molecular types distribution & $17(10 \%)$ & $23(14 \%)$ & $32(20 \%)$ & $90(56 \%)$ \\
\hline Total & $13(13 \%)$ & $17(17 \%)$ & $17(17 \%)$ & $52(53 \%)$ \\
\hline Partial & $4(6 \%)$ & $6(10 \%)$ & $15(24 \%)$ & $38(60 \%)$ \\
\hline p-value & \multicolumn{4}{|c|}{0.21} \\
\hline
\end{tabular}


Table II. Cont.

\begin{tabular}{|c|c|c|c|c|}
\hline & \multicolumn{4}{|c|}{ MOLECULAR TYPE } \\
\hline & Classical & MesenChyMal & Proneural & Neural \\
\hline \multicolumn{5}{|l|}{ Chemotherapy $(\mathrm{n}=162)$} \\
\hline Molecular types distribution & $17(10 \%)$ & $23(14 \%)$ & $32(20 \%)$ & $90(56 \%)$ \\
\hline Used & $3(9 \%)$ & $6(18 \%)$ & $3(9 \%)$ & $21(64 \%)$ \\
\hline Not used & $14(11 \%)$ & $17(13 \%)$ & $29(22 \%)$ & $69(53 \%)$ \\
\hline $\mathrm{p}$-value & \multicolumn{4}{|c|}{0.33} \\
\hline \multicolumn{5}{|l|}{ Radiation therapy $(\mathrm{n}=162)$} \\
\hline Molecular types distribution & $17(10 \%)$ & $23(14 \%)$ & $32(20 \%)$ & $90(56 \%)$ \\
\hline Used & $3(9 \%)$ & $5(14 \%)$ & $12(34 \%)$ & $15(43 \%)$ \\
\hline Not used & $14(11 \%)$ & $18(14 \%)$ & $20(16 \%)$ & $75(59 \%)$ \\
\hline $\mathrm{p}$-value & \multicolumn{4}{|c|}{0.56} \\
\hline \multicolumn{5}{|l|}{ Overall survival $(\mathrm{n}=162)$} \\
\hline Molecular types distribution & $17(10 \%)$ & $23(14 \%)$ & $32(20 \%)$ & $90(56 \%)$ \\
\hline Minimum & 40 & 49 & 21 & 4 \\
\hline Maximum & 1234 & 1365 & 1814 & 2837 \\
\hline Median & 319 & 299 & 188.5 & 238.5 \\
\hline SD & 128.5 & 249.75 & 282 & 231.5 \\
\hline p-value & \multicolumn{4}{|c|}{0.6} \\
\hline \multicolumn{5}{|l|}{ Relapse free survival $(\mathrm{n}=39)$} \\
\hline Molecular types distribution & $5(13 \%)$ & $5(13 \%)$ & $8(20 \%)$ & $21(54 \%)$ \\
\hline Minimum & 34 & 75 & 28 & 20 \\
\hline Maximum & 258 & 288 & 999 & 1070 \\
\hline Median & 88 & 154 & 199 & 108 \\
\hline SD & 70.25 & 113 & 197 & 52 \\
\hline $\mathrm{p}$-value & \multicolumn{4}{|c|}{0.6} \\
\hline
\end{tabular}

tumor diameter was recorded in patients with GBM proneural type $($ median $=2.55 \mathrm{~cm}, \mathrm{SD}=0.35)$, and the lowest in mesenchymal tumors (median $=1.95$ $\mathrm{cm}, \mathrm{SD}=0.55)$. Both the smallest $(0.5 \mathrm{~cm})$ and the largest $(4.4 \mathrm{~cm})$ tumor diameter were recorded among neural tumors. In the analyzed locations, the most common were neural tumors, accounting for $59 \%$ of all tumors in the right-sided location, $53 \%$ of all tumors in the left-sided location and $50 \%$ of all tumors in the bilateral location. In the left-sided and right-sided location, classical tumors were the least numerous, accounting for $11 \%$ of the total in each of these locations. In the GBM with bilateral localization group no classical tumors were found at all. In each molecular type, the vast majority were primary tumors. Most of them were found among neural type tumors (55\%). On the other hand, the lowest number was found among classical type $(11 \%)$. The highest number of secondary tumors was noted in the neural type $(75 \%)$, while in the classical and mesenchymal types were not found at all. The vast majority of neoplastic tumors were monofocal (95\%). The highest number of monofocal tumors was recorded among neural-type tumors (56\%), and the lowest among classic-type tumors (10\%). Among multifocal tumors, which accounted for $5 \%$ of the total, their number was the highest in the group of neural type tumors (50\%), and the lowest in the group of classic and proneural type tumors $(12.5 \%)$. Most of the tumors were completely resected $(61 \%)$. Most of them were recorded in the neural type (53\%), and the least in the classical type (13\%). Among partially resected tumors, the trend was similar, with 6\% classical and 60\% neural type. Most patients $(80 \%)$ did not receive CTH. Among them, the greatest number were those with neural type tumors $(53 \%)$, and the fewest were those with classical type tumors (11\%). Among patients who underwent $\mathrm{CTH}$, the greatest number were those with neural type tumors (64\%), and the fewest were those with classical and proneural type tumors (9\%). Most patients $(78 \%)$ did not receive $\mathrm{RTH}$. Of these, the most 
had tumors of the neural type (59\%), and the fewest had tumors of the classic type (11\%). Among patients in whom RTH was applied, the tendency was similar, and the most were also those with neural type tumors (43\%), and the fewest had classic type tumors (9\%). The highest median overall survival was seen in patients diagnosed with the classical type of tumor (median $=319$ days, SD $=128.5)$, while the lowest one was in patients with the proneural type of tumor (median $=188.5$ days, $\mathrm{SD}=282)$. Both the lowest (4 days) and the highest (2,837 days) number of days lived were recorded among patients with the neural type tumors. Analysis performed for 39 patients with documented recurrence of the neoplastic disease showed that the highest median relapse-free survival was in patients diagnosed with the mesenchymal type of tumor (median $=154$ days, SD = 113), while the lowest was in patients with the classic type (median $=88$ days, $\mathrm{SD}=70.25)$. Both the lowest (20 days) and the highest (1070 days) number of relapse-free days were recorded among patients with the neural tumor type. The relationship between tumor types and clinical and morphological parameters is presented in Table II.

\section{Survival analysis}

Kaplan-Meier analysis showed no statistically significant correlations between the GBM tumor molecular type and overall survival $(\mathrm{p}=0.98)$. The results are presented in Fig. 3. Using the Cox proportional hazards model, a statistically significant relationship between the overall survival time and all covariates demonstrated in the model was found $\left(\chi^{2}=22.36\right.$, $\mathrm{DF}=12, \mathrm{p}=0.04)$. The statistical significance of the model was determined by 3 determinants: tumor

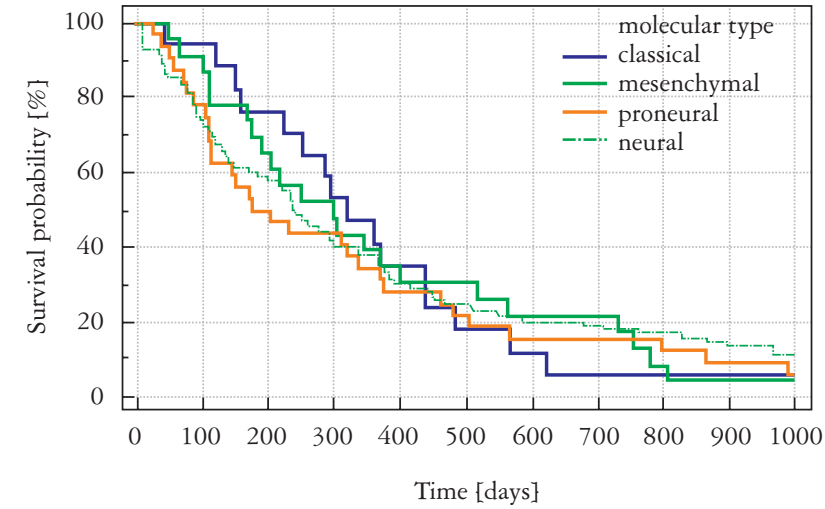

Fig. 3. Overall survival depending on the GBM molecular type $(\mathrm{n}=162)$

location (HR 2.79, 95\% CI: 1.03-7.59, p=0.04) extent of surgical resection (HR 2.08, 95\% CI: $1.33-$ 3,26, $\mathrm{p}=0.001$ ) and patient's age at diagnosis of GBM (HR 1.02, 95\% CI: 1.00-1.04, p=0.02). The results are presented in Table III.

\section{Results summary}

No significant relationship was observed between the molecular type of GBM tumors and the studied clinical and morphological parameters of prognostic significance. There were also no statistically significant correlations between the GBM tumor molecular type and survival, both in terms of overall survival and relapse free survival. Analyzing the impact of all prognostic variables and molecular type of GBM on the probability of overall survival, statistically significant relationships were found. The statistical significance of the model was determined by 3 deter-

Table III. Cox proportional hazards model - overall survival $(\mathrm{n}=162)$

\begin{tabular}{lcccc}
\hline Parameters & WaLD & P-VALUE & $\boldsymbol{\beta}$ HR & 95\% CI OF $\boldsymbol{\beta}$ HR \\
\hline Use of radiation therapy & 1.56 & 0.21 & 0.72 & $0.43-1.21$ \\
\hline Multifocal tumor & 0.005 & 0.95 & 1.04 & $0.35-3.05$ \\
\hline Secondary tumor & 2.61 & 0.11 & 5.78 & $0.69-48.45$ \\
\hline Mesenchymal tumor type & 0.001 & 0.97 & 1.02 & $0.42-2.44$ \\
\hline Proneural tumor type & 1.25 & 0.26 & 1.54 & $0.72-3.23$ \\
\hline Neural tumor type & 0.0005 & 0.98 & 0.99 & $0.51-1.92$ \\
\hline Male gender & 0.85 & 0.36 & 0.82 & $0.55-1.24$ \\
\hline Tumor diameter (cm) & 0.39 & 0.53 & 0.91 & $0.68-1.22$ \\
\hline Bilateral tumor & 4.03 & 0.04 & 2.79 & $1.03-7.59$ \\
\hline Right-sided tumor & 2.05 & 0.15 & 1.37 & $0.89-2.12$ \\
\hline Age at diagnosis & 5.78 & 0.02 & 1.02 & $1.00-1.04$ \\
\hline Partial resection & 10.30 & 0.001 & 2.08 & $1.33-3.26$ \\
\hline Partial resection & 1.0089 & 0.32 & 0.77 & $0.46-1.28$ \\
\hline
\end{tabular}


minants: tumor location, extent of surgical resection and the patient's age at the diagnosis of GBM.

\section{Discussion}

To determine molecular markers of GBM tumors, no expensive and complicated methods of studying gene expression are needed. In clinical conditions a generally available and cheaper method of identifying immunohistochemical markers in GBM tumor material embedded in paraffin blocks can be proposed. Molecular markers of glioblastoma, the algorithms for classifying tumors into specific molecular types and even the types themselves have changed over the years $\{6,7,8,9,10,11\}$. In this study, six proteins were used as molecular markers of glioblastoma, i.e. EGFR, PDGFRA, NF1, IDH1, p53 and PTEN, which allowed us to classify GBM tumors into four molecular types - classical, mesenchymal, proneural and neural - according to the classification proposed by Verhaak et al. [6]. In later years, Le Mercier $e t$ al. divided GBM tumors into two main molecular types: "proneural-like" and "classical-like". Tumors that did not qualify for either type were termed "other". The following proteins were selected as molecular markers: EGFR, PDGFRA and p53 [9]. Chaurasia et al. divided GBM tumors into three groups, using the immunohistochemical expression of three proteins: ATRX, p53 and IDH1. As a result, three groups of gliomas were obtained: ATRX/ IDH1, ATRX/p53, IDH1/p53 [10]. Nagy et al. suggested establishing affiliation to one of four molecular types (classical, mesenchymal, proneural, neural) based on the analysis of the expression of another 3 proteins: IDH1, EGFR and NF1 [11]. Despite numerous studies, currently the only officially accepted markers of GBM tumors are: $M G M T$ gene promoter methylation, IDH1/2 R132H mutation and $1 \mathrm{p} / 19 \mathrm{q}$ codeletion, although the latter mainly informs whether there is a oligodendroglioma component in the examined tumor. In the new classification of central nervous system tumors (2016), WHO distinguished 2 types of GBM tumors: IDH1 wild type (90\% of GBM tumors, the most common in older people with primary tumors) and IDH1 mutant (10\% of GBM tumors, usually secondary tumors in young people). The most common point mutation in the IDH1 gene is the $R 132 \mathrm{H}$ mutation, on the basis of which the above-mentioned WHO classification was created. IDH1-mutant tumors are tumors of the proneural type [12, 13]. As in this study, Le Mercier et al. did not detect statistically significant relationships between the GBM molecular type and age of cancer diagnosis, sex, monofocal vs. multifocal tumor character, extent of surgical resection and type of treatment (RTH/CTH); however, similar to this work, as independent factors together, they signifi- cantly influenced the patients overall survival. On the other hand, they observed a statistically significant relationship between the patients' overall survival and the molecular type of glioblastoma, determining the chance of survival of patients with proneural type tumors as the highest. Another difference between their work and this study was that most of the tumors they studied were proneural type. However, the division presented by them did not take into account the mesenchymal and neural types, which were the most numerous in this work [9]. Also, Nagy et al. did not find any statistically significant relationships between the molecular type of glioblastoma and the age of diagnosis, sex and overall survival. The ratio of primary and secondary tumors in their work was also close to that observed by us. However, they noted a longer mean survival and an earlier age of GBM onset among patients whose cancer cells showed the presence of the IDH1 R132H mutation. In this work, we did not directly assess the relationship between the presence of the IDH1 $\mathrm{R} 132 \mathrm{H}$ mutation and clinical parameters of prognostic significance. According to the above-mentioned authors, the most common type of tumor was the classic type, while the neural type was the least frequent [11]. Also Verhaak et al. noted the highest percentage of classical type tumors and the lowest of neural type [6]. Jakovlevs et al. in their study were assessed whether gliomas could be subdivided into different molecular subtypes by immunohistochemistry. For this purpose they tested GBM tumors for PDGFRA, IDH1 R132H, CD44, p53, Ki-67, p21 and p27 expression and subdevided them into 3 molecular types: proneural, mesenchymal and not otherwise classified. In their study the highest percentage were proneural tumors, in our case they were neural tumors, which the authors of the above study did not distinguish. Like in our study, no prognostic role was found for the molecular subtypes, but as opposed to our results predictive roles were noted. Also different than in our study both proneural and mesenchymal molecular subtypes showed a benefit from the addition of chemotherapy and radiotherapy. However, it should be borne in mind that both the selection of markers and the division into molecular groups of tumors differed in this work compared to ours [14]. Tyler $e t$ al. reported that tumors of proneural and neural types are more often located in the left hemisphere of the brain, in particular in the frontotemporal area. Classical type tumors, on the other hand, are characterized by a more diffuse type of growth. In this study, we did not note any statistically significant relationship between the molecular type of the tumor and its location, although our data referred only to the hemispheres of the brain, not to a specific location. The inability to determine the exact location of individual tumors was due to the lack of standard- 
ized terminology used by the operators and the lack of such data in the history of the disease [15]. Many factors can contribute to the divergent results. The first one may be the selection and characteristics of the study group. The group of patients recruited for the study is only a fragment of the entire population of people with GBM tumors. The problem may also be in the size of some groups, when we analyzed a specific factor, e.g. tumor unifocal/multifocal and primary/secondary character, where the number of cases was far too low. The choice of treatment, which was compliant with the current therapeutic standards, may show some individual differences. This is due to the fact that the patient's condition (side effects of the treatment, coexisting diseases, tumor localization) sometimes necessitates the individualization of the therapeutic procedure. Aubry et al. identified a relationship between the molecular type of glioblastoma and the location of the tumor biopsy. In the material from the tumor boundaries, proneural and neural types of GBM tumors were noted more often. On the other hand, the material collected from the foci of necrosis and the central part of the tumor correlated more often with classical and mesenchymal type. The above fact is extremely important in relation to the tissue microarray technique used in this work. As a result, only selected tumor fragments were analyzed, which could have influenced its affiliation to a specific molecular type [16]. Another possible factor affecting the results, is the differences in tested proteins expression patterns due to accompanying genetic abnormalities, e.g. some even minor deletions and insertions. Many of them may affect not only the proteins expression, but also their intracellular localization and detection in IHC reactions $[11,17,18,19,20]$. What else could have affected the results of the study is omitting the role of molecular marker of oligodendroglioma $-1 \mathrm{p} / 19 \mathrm{q}$ codeletion and methylation patterns in determining the relationship between the molecular and clinical specification of the studied tumors. Both the G-CIMP tumor phenotype and methylation of the MGMT gene promoter are associated with longer survival and better response to treatment, as well as presence of $1 \mathrm{p} / 19 \mathrm{q}$ codeletion $[21,22,23,24,25]$. Another factor influencing the results is the evolution of cancer cells. These changes may result from positive selection in response to therapeutic treatment, i.e. the treatment will destroy sensitive cells and leave resistant cells. It is one of the key factors contributing to therapeutic failure and drug resistance [21, 26, 27]. Also the presence of stem cells could be the reason of GBM tumors hereogeneity. Glioblastoma stem cells (GSC) are cells capable of self-renewal, showing pluripotency, the ability to proliferate, angiogenesis, invade and modulate the immune response. Due to the above characteristics, they may be responsible for in- tra-tumor cell heterogeneity and resistance to treatment $[21,28,29]$. What we should not forget when talking about the GBM tumors heterogeneity is its microenvironment. It affects both the patients' response to treatment and their survival. Various factors including infiltrating stroma, blood vessels, secreted factors, immune cells and surrounding matrix together influence tumor progression, invasion, and eventual recurrence. The microenvironment also has an impact on the emergence and evolution of genetic abnormalities underlying the classification of GBM tumors into particular molecular types. It also modulates the host's immune response $[21,30,31,32]$. Analyzing the results of the this study and the facts presented in the discussion current knowledge about the molecular basis of gliomas and factors affecting their development does not allow us to classify them solely on the basis of classical molecular subtypes. Therefore, it is also difficult to establish true relationships between such classifications and prognostic factors. In the future, the tools used to classify gliomas and to search for dedicated therapeutic goals must therefore be constructed in a way that allows for consideration of both the broadly understood molecular basis of GBM tumors (including their heterogeneity) and the microenvironment affecting them.

\section{The authors declare no conflict of interest.}

\section{References}

1. Velásquez C, Mansouri S, Mora C, et al. Molecular and clinical insights into the invasive capacity of glioblastoma cells. J Oncol 2019; 1: 740-763.

2. Batash R, Asna N, Schaffer P, et al. Glioblastoma multiforme, diagnosis and treatment; recent literature review. Curr Med Chem 2017; 24: 3002-3009.

3. Zhou W, Wahl DR. Metabolic abnormalities in glioblastoma and metabolic strategies to overcome treatment resistance. Cancers 2019; 11: 1231.

4. Gately L, McLachlan SA, Philip J, et al. Molecular profile of long-term survivors of glioblastoma: a scoping review of the literature. Clin Neurosci 2019; 68: 1-8.

5. Noch EK, Ramakrishna R, Magge R. Challenges in the treatment of glioblastoma: multisystem mechanisms of therapeutic resistance. World Neurosurg 2018; 116: 505-517.

6. Verhaak RG, Hoadley KA, Purdom E, et al. Integrated genomic analysis identifies clinically relevant subtypes of glioblastoma characterized by abnormalities in PDGFRA, IDH1, EGFR and NF1. Cancer Cell 2010; 17: 98-110.

7. Cancer Genome Atlas Research Network, Comprehensive genomic characterization defines human glioblastoma genes and core pathways. Nature 2008; 455: 1061-1068.

8. Brennan CW, Verhaak RG, McKenna A, et al. The somatic genomic landscape of glioblastoma. Cell 2013; 155: 462-477.

9. Le Mercier M, Hastir D, Lopez XM, et al. A simplified approach for the molecular classification of glioblastomas. PLoS One 2012; 7: e45475.

10. Chaurasia A, Park SH, Seo JW, et al. Immunohistochemical analysis of ATRX, IDH1 and p53 in glioblastoma and their correlations with patient survival. J Korean Med Sci 2016; 31 : 1208-1214. 
11. Nagy Á, Garzuly F, Padányi G, et al. Molecular subgroups of glioblastoma - an assessment by immunohistochemical markers. Pathol Oncol Res 2019; 25: 21-31.

12. Jovčevska I. Genetic secrets of long-term glioblastoma survivors. Bosn J Basic Med Sci 2019; 19: 116-124.

13. Wesseling P, Capper D. WHO 2016 Classification of gliomas. Neuropathol Appl Neurobiol 2018; 44: 139-150.

14. Jakovlevs A, Vanags A, Gardovskis J, et al. Molecular classification of diffuse gliomas. Pol J Pathol 2019; 70: 246-258.

15. Steed TC, Treiber JM, Patel K, et al. Differential localization of glioblastoma subtype: implications on glioblastoma pathogenesis. Oncotarget 2016; 7: 24899-24907.

16. Aubry M, de Tayrac M, Etcheverry A, et al. From the core to beyond the margin: a genomic picture of glioblastoma intratumor heterogeneity. Oncotarget 2015; 6: 12094-12109.

17. McKeever PE, Dennis TR, Burgess AC, et al. Chromosome break point at $17 \mathrm{q} 11.2$ andinsertion of DNA from three different chromosomes in a glioblastoma with exceptional glial fibrillary acidic protein expression. Cancer Genet Cytogenet 1996; 87: 41-47.

18. Vandenbroucke I, Van Oostveldt P, Coene E, et al. Neurofibromin is actively transported to the nucleus. FEBS Lett 2004; 560: 98-102.

19. Felsberg J, Hentschel B, Kaulich K, et al. Epidermal Growth Factor Receptor Variant III (EGFRvIII) Positivity in EGFR-amplified glioblastomas: prognostic role and comparison between primary and recurrent tumors. Clin Cancer Res 2017; 23: 6846-6855.

20. Karnam S, Kottu R, Chowhan AK, et al. Expression of p53 \& epidermal growth factor receptor in glioblastoma. Indian J Med Res 2017; 146: 738-745.

21. Lee E, Yong RL, Paddison P, et al. Comparison of glioblastoma (GBM) molecular classification methods. Semin Cancer Biol 2018; 53: 201-211

22. Noushmehr H, Weisenberger DJ, Diefes K, et al. Identification of a $\mathrm{CpG}$ island methylator phenotype that defines a distinct subgroup of glioma. Cancer Cell 2010; 17: 510-522.

23. Brennan CW, Verhaak RG, McKenna A, et al. The somatic genomic landscape of glioblastoma. Cell 2013; 155: 462-477.

24. He ZC, Ping YF, Xu SL, et al. Fetal lower MGMT expression predicts better prognosis in proneural-like glioblastoma. Int J Clin Exp Med 2015; 8: 20287-20294.

25. Myung JK, Jin Cho H, Kim H, et al. Prognosis of glioblastoma with oligodendroglioma component is associated with the IDH1 mutation and MGMT methylation status. Transl Oncol 2014; 7: 712-719.

26. Sottoriva A, Spiteri I, Piccirillo SG, et al. Intratumor heterogeneity in human glioblastoma reflects cancer evolutionary dynamics. Proc Natl Acad Sci U S A 2013; 110: 4009-4014.

27. Johnson BE, Mazor T, Hong C, et al. Mutational analysis reveals the origin and therapy-driven evolution of recurrent glioma. Science 2014; 343: 189-193.

28. Kalkan R. Glioblastoma stem cells as a new therapeutic target for glioblastoma. Clin Med Insights Oncol 2015; 9: 95-103.

29. Lan X, Jörg DJ, Cavalli FMG, et al., Fate mapping of human glioblastoma reveals an invariant stem cell hierarchy. Nature 2017; 549: 227-232.

30. Eder K, Kalman B. Molecular heterogeneity of glioblastoma and its clinical relevance. Pathol Oncol Res 2014; 20: 777-787.

31. Severson B, Li E, Pignon JC, et al. Comprehensive analyses of tumor immunity: implications for cancer immunotherapy. Genome Biol 2016; 17: 174.

32. Huang B, Zhang H, Gu L, et al. Advances in immunotherapy for glioblastoma multiforme. J Immunol Res 2017; 3: 597-613.

\section{Address for correspondence}

Sylwia Jankowska

Department of Pathology

Pomeranian Medical University

Rybacka 1

70-204 Szczecin, Poland

e-mail: sylwia_jankowska@interia.eu 